\title{
Automatic Image Segmentation Using Active Contours with Univariate Marginal Distribution
}

\author{
I. Cruz-Aceves, ${ }^{1}$ J. G. Avina-Cervantes, ${ }^{1}$ J. M. Lopez-Hernandez, ${ }^{1}$ M. G. Garcia-Hernandez, \\ M. Torres-Cisneros, ${ }^{1}$ H. J. Estrada-Garcia, ${ }^{1}$ and A. Hernandez-Aguirre ${ }^{2}$ \\ ${ }^{1}$ División de Ingenierías Campus Irapuato-Salamanca, Universidad de Guanajuato, Carretera Salamanca-Valle de \\ Santiago Km 3.5+1.8 Km Comunidad de Palo Blanco, 36885 Salamanca, GTO, Mexico \\ ${ }^{2}$ Centro de Investigación en Matemáticas (CIMAT), A.C. Jalisco S/N, Col. Valenciana, 36000 Guanajuato, GTO, Mexico
}

Correspondence should be addressed to I. Cruz-Aceves; i.cruzaceves@ugto.mx

Received 19 July 2013; Accepted 23 October 2013

Academic Editor: Marco Perez-Cisneros

Copyright (C) 2013 I. Cruz-Aceves et al. This is an open access article distributed under the Creative Commons Attribution License, which permits unrestricted use, distribution, and reproduction in any medium, provided the original work is properly cited.

\begin{abstract}
This paper presents a novel automatic image segmentation method based on the theory of active contour models and estimation of distribution algorithms. The proposed method uses the univariate marginal distribution model to infer statistical dependencies between the control points on different active contours. These contours have been generated through an alignment process of reference shape priors, in order to increase the exploration and exploitation capabilities regarding different interactive segmentation techniques. This proposed method is applied in the segmentation of the hollow core in microscopic images of photonic crystal fibers and it is also used to segment the human heart and ventricular areas from datasets of computed tomography and magnetic resonance images, respectively. Moreover, to evaluate the performance of the medical image segmentations compared to regions outlined by experts, a set of similarity measures has been adopted. The experimental results suggest that the proposed image segmentation method outperforms the traditional active contour model and the interactive Tseng method in terms of segmentation accuracy and stability.
\end{abstract}

\section{Introduction}

Automatic image segmentation is an important and challenging problem in computer vision and medical image analysis. The objective of image segmentation is to separate objects of interest from a given image based on different attributes such as shape, color, intensity, or texture. In recent years, several techniques have been reported for this purpose including graph cut $[1,2]$, improved watershed transform [3], suppressed fuzzy c-means [4], supervised fuzzy clustering [5], multithreshold based on differential evolution [6], and active contour model (ACM), which has been applied in different areas such as intravascular ultrasound images [7], automatic urban buildings [8], and natural images [9], to name a few.

The Active Contour Model is an energy-minimizing spline curve composed of discrete control points called snaxels. The curve is attracted towards features as edges of a target object through the evaluation of internal and external forces. The classical implementation of ACM is prone to be trapped into local minima problem and it is also highly sensitive to initialization of the control points because they require being close to the target object; otherwise failure of convergence will occur.

Since ACM was introduced by [10], many researchers have suggested adapting different techniques to work together with ACM in order to overcome its shortcomings. The suggested improvements of the classical ACM including the introduction of prior knowledge such as active shape models [11], shape prior applied on human cerebellum [12], ACM based on level set method [13], populationbased methods such as genetic algorithms [14], differential evolution [15], and particle swarm optimization [16]. The performance of these population-based methods working together with ACM is robust in local minima problem and according to the tests, these methods present a more stable and efficient image segmentation within an appropriate computational time. 
The population-based methods are an effective way to solve discrete optimization problems. Recently, a new approach known as Estimation of Distribution Algorithms (EDAs) from the family of Evolutionary Algorithms has begun to attract more attention for solving global optimization problems with a fast convergence. EDAs are stochastic methods that incorporate statistical knowledge to solve optimization problems [17]. These algorithms consist of a set of potential solutions called population, where each potential solution is referred to as individual, and in general, EDAs work with truncation selection and binary encoding to explore the search space. In the EDAs strategy a subset of individuals is selected, and a probabilistic model of these individuals is constructed. The new individuals will be generated from this model, and the algorithm evolves until a stopping criterion is satisfied. Since EDAs are highly efficient in solving optimization problems, they have been successfully applied in a wide range of applications such as the side chain placement problem [18], dynamic optimization [19], cancer chemotherapy optimization [20], and multiobjective knapsack problem [21].

In this paper, we introduce a novel automatic image segmentation method based on the theory of Active Contour Model and Estimation of Distribution Algorithms. The proposed method uses the Univariate Marginal Distribution Algorithm (UMDA) to infer statistical dependencies between snaxels belonging to a population, in order to increase the exploration and exploitation capabilities regarding the classical Active Contour Model. To establish the initial positioning of the proposed method, a shape prior and the alignment process proposed in [22] to construct a target object template are used. This template is discretized and it is also scaled to different size in order to generate the initial populations of individuals and assuming that the target object is confined within them. This proposed method addresses the problem of segmenting the hollow core in microscopic images of photonic crystal fibers and the human heart and ventricular areas from Computed Tomography and Magnetic Resonance images, respectively.

The remainder of this paper is organized as follows. In Section 2, the fundamentals of Active Contour Model and Univariate Marginal Distribution Algorithm are introduced. In Section 3, the proposed image segmentation method is presented, along with a set of similarity metrics to evaluate its performance. The experimental results are discussed in Section 4, and conclusions are given in Section 5.

\section{Background}

In this section, the fundamentals of the Active Contour Model and the Univariate Marginal Distribution Algorithm are described in detail.

2.1. Active Contour Models. The traditional Active Contour Model, also known as snake, is a parametric curve that can move within a spatial image domain where it was defined [10]. The curve is defined by $p(s, t)=(x(s, t), y(s, t)), s \in[0,1]$, where it evolves through time $t$ to minimize the total energy function given by the following:

$$
E_{\text {snake }}=\int_{0}^{1}\left[E_{\text {int }}(p(s, t))+E_{\text {ext }}(p(s, t))\right] d s .
$$

This energy function is composed of two different energies: the external energy $E_{\text {ext }}$ defined by the particular gradient features of the image, and the internal energy $E_{\text {int }}$, which is used to control the shape modification of the curve and to maintain the search within the spatial image domain. On the other hand, the discrete computational implementation of the classical ACM consists of a set of $n$ discrete points $\left\{p_{i} \mid i=1,2, \ldots, n\right\}$, and the local energy function is given by (2), which is iteratively evaluated in order to minimize, for the actual discrete point, the $k_{i}$ index in the $W_{i}$ searching window using (3):

$$
\begin{aligned}
E_{i, j} & =E_{\text {int }}+E_{\text {ext }}, \\
E_{\text {snake }}=\sum_{i=1}^{n} E_{i, k_{i}}, \quad k_{i} & =\arg \min _{j}\left(E_{i, j}\right), \quad j \in W_{i} .
\end{aligned}
$$

Since the classical ACM has the drawbacks of initialization and local minima problem, Chan and Vese [13] incorporated a shape prior constraint within the traditional ACM. This method is defined by using the following:

$$
E_{T}=w_{1} E_{1}+w_{2} E_{2}+w_{3} E_{3} .
$$

$E_{T}$ represents the total energy function composed of the energies $E_{1}, E_{2}$, and $E_{3}$ with their weighting factors $w_{1}, w_{2}$, and $w_{3}$, where $E_{1}$ represents the active contour or snake. The energy $E_{2}$ represents the shape energy defined by the difference between the active contour and the shape template and it is expressed as follows:

$$
E_{2}=\int_{\Omega}\left(H(\phi)-H\left(\varphi_{T}\left(B^{T}\right)\right)\right)^{2} d x d y
$$

where $\Omega$ is the image domain, $H(\cdot)$ is the Heaviside function, $\phi$ is a signed distance function, $\varphi_{T}$ the deformed template, and $B^{T}$ the transformation matrix consisting of translation $\left[t_{x}, t_{y}\right]^{T}$, scaling $[s]$, and rotation $[\theta]$ parameters, as follows:

$$
B^{T}=\underbrace{\left[\begin{array}{ccc}
1 & 0 & t_{x} \\
0 & 1 & t_{y} \\
0 & 0 & 1
\end{array}\right]}_{M(a, b)} \times \underbrace{\left[\begin{array}{lll}
s & 0 & 0 \\
0 & s & 0 \\
0 & 0 & 1
\end{array}\right]}_{H(s)} \times \underbrace{\left[\begin{array}{ccc}
\cos \theta & -\sin \theta & 0 \\
\sin \theta & \cos \theta & 0 \\
0 & 0 & 1
\end{array}\right]}_{R(\theta)},
$$

where $t_{x}$ and $t_{y}$ are the translation parameters in the horizontal and vertical axes, respectively. ( $s)$ is the scaling factor, and $\theta$ represents the rotation angle parameter. Finally, the third energy $E_{3}$ is the image-based force with an image intensity $I$ and the gradient operator $\nabla$ computed as follows:

$$
E_{3}=\int_{\Omega}(\nabla H(\phi)-\nabla I)^{2} d x d y .
$$

These three energies are iteratively evaluated, until the difference between the previous and current segmented 
area becomes stable. Although the Chan and Vese method is suitable to solve the initialization disadvantage of the classical ACM, this method is prone to be trapped into local minima. An alternative to overcome this drawback is to use population-based methods such as Estimation of Distribution Algorithms, which are described in the following Section 2.2.

\subsection{Overview of Estimation of Distribution Algorithms} (EDAs). Estimation of Distribution Algorithms [23-25] are population-based stochastic algorithms that incorporate statistical information to solve optimization problems. Although EDAs are based on the principles of evolutionary computation since they use a population of individuals, binary encoding, and selection operators, EDAs replace the application of the crossover and mutation operators by building probabilistic models of promising solutions based on global statistical information. The probabilistic model that EDAs build in each generation is designed to infer statistical dependencies between the variables in order to generate new solutions. In our work, we will focus on the Univariate Marginal Distribution Algorithm, which is one of EDAs that works perfectly for linear problems and for applications without many significant dependencies [26, 27]. UMDA works on binary strings and uses a probability vector $\mathbf{p}=\left(p_{1}, p_{2}, \ldots, p_{n}\right)^{T}$ to construct the probabilistic model and then create new solutions for each variable independently, where $p_{i}$ represents the probability of obtaining a 1 (binary encoding) in position $i$. The main idea behind UMDA is that it approximates the actual probability distribution of the individuals in $\mathbb{P}_{t}$ as the product of the univariate frequencies calculated from the selected population and assuming that all variables are independent [28]. In general, UMDA iterates the steps of selection, estimation of probability distribution and the creation of new individuals. In the first step, after the individuals in the search space $\Omega$ have been sorted according to fitness, the selection probability $s$ is calculated by using the following proportional selection:

$$
\mathbb{P}^{s}(x)=\frac{\mathbb{P}(x) f(x)}{\sum_{\tilde{x} \in \Omega} \mathbb{P}(\tilde{x}) f(\tilde{x})} .
$$

In the second step a joint probability $\mathbb{P}$ is computed through the following:

$$
\mathbb{P}(x)=\prod_{i=1}^{n} \mathbb{P}\left(X_{i}=x_{i}\right),
$$

where $x=\left(x_{1}, x_{2}, \ldots, x_{n}\right)^{T}$ is the binary value of the $i$ th bit in the binary string (chromosome) and $X_{i}$ represents the $i$ th component of the random vector $X$. The last step of UMDA generates new individuals from the estimated distribution which will be evaluated according to the fitness function in the next generation. These three steps are iteratively performed until the termination criteria are satisfied.

According to the above description, the UMDA algorithm can be implemented through the following.

(1) Establish $t=0$. Generate $n$ individuals randomly.

(2) Select a subpopulation $S$ of $m \leq n$ individuals according to a selection method.
(3) Compute the univariate marginal probabilities $p_{i}^{s}\left(x_{i}, t\right)$ of $S$.

(4) Generate $n$ new individuals according to $p(x, t+1)=$ $\prod_{i=1}^{n} p_{i}^{s}\left(x_{i}, t\right)$.

(5) Stop if the convergence criterion is satisfied (e.g., stability or number of generations); otherwise, repeat steps (2)-(5).

\section{Proposed Image Segmentation Method}

The proposed image segmentation method based on Active Contour Model and the Univariate Marginal Distribution Algorithm is described in Section 3.1. Additionally, to assess the performance of the proposed method, the Jaccard and Dice indices are explained in Section 3.2.

3.1. Active Contour Model with Univariate Marginal Distribution. Due to the two main shortcomings of the traditional ACM discussed above, the Univariate Marginal Distribution Algorithm has been adopted to solve the local minima drawback by building probabilistic models, and the initialization disadvantage is addressed by using scaled templates obtained from an alignment process. Since the methodology of the proposed method makes it possible to apply the UMDA strategy directly in the segmentation task, the advantages of low computational time, efficiency, and robustness are inherently acquired. The procedure of the proposed method is illustrated in Figure 1, and it is described below. This procedure is similar to [15] in the final segmentation step, while the main difference is the introduction of shape templates avoiding the user interaction via seed point and also the use of an Euclidean distance parameter between individuals instead of constrained polar sections.

The first step of the proposed method consists of the construction of a shape template through the alignment of a training set of selected reference images, which leads to differences in position, direction, and scale. The alignment process is performed using the technique developed in [22], by estimating the parameters $[a, b, s, \theta]^{T}$ as follows:

$$
\left[\begin{array}{c}
\tilde{x} \\
\tilde{y} \\
1
\end{array}\right]=M(a, b) \times H(s) \times R(\theta) \times\left[\begin{array}{l}
x \\
y \\
1
\end{array}\right],
$$

where $M(a, b)$ is the $x$ (horizontal) and $y$ (vertical) translation matrix, $H(s)$ is the scale matrix, and $R(\theta)$ is the rotation matrix. The product of the three matrices maps the coordinates $(x, y) \in \mathbb{R}^{2}$ to coordinates $(\tilde{x}, \tilde{y}) \in \mathbb{R}^{2}$, which is used to apply the gradient descent method iteratively in order to minimize the following energy function:

$$
E_{\mathrm{alig}}=\sum_{i=1}^{n} \sum_{j=1, j \neq i}^{n}\left\{\frac{\iint_{\Omega}\left(\widetilde{I}^{i}-\widetilde{I}^{j}\right)^{2} d A}{\iint_{\Omega}\left(\widetilde{I}^{i}+\widetilde{I}^{j}\right)^{2} d A}\right\},
$$

where $\Omega$ is the image domain and $\widetilde{I}$ is the transformed image. The final process of this step involves obtaining the final aligned template by superimposing all transformed images and then acquires it through the maximum shape boundary. 

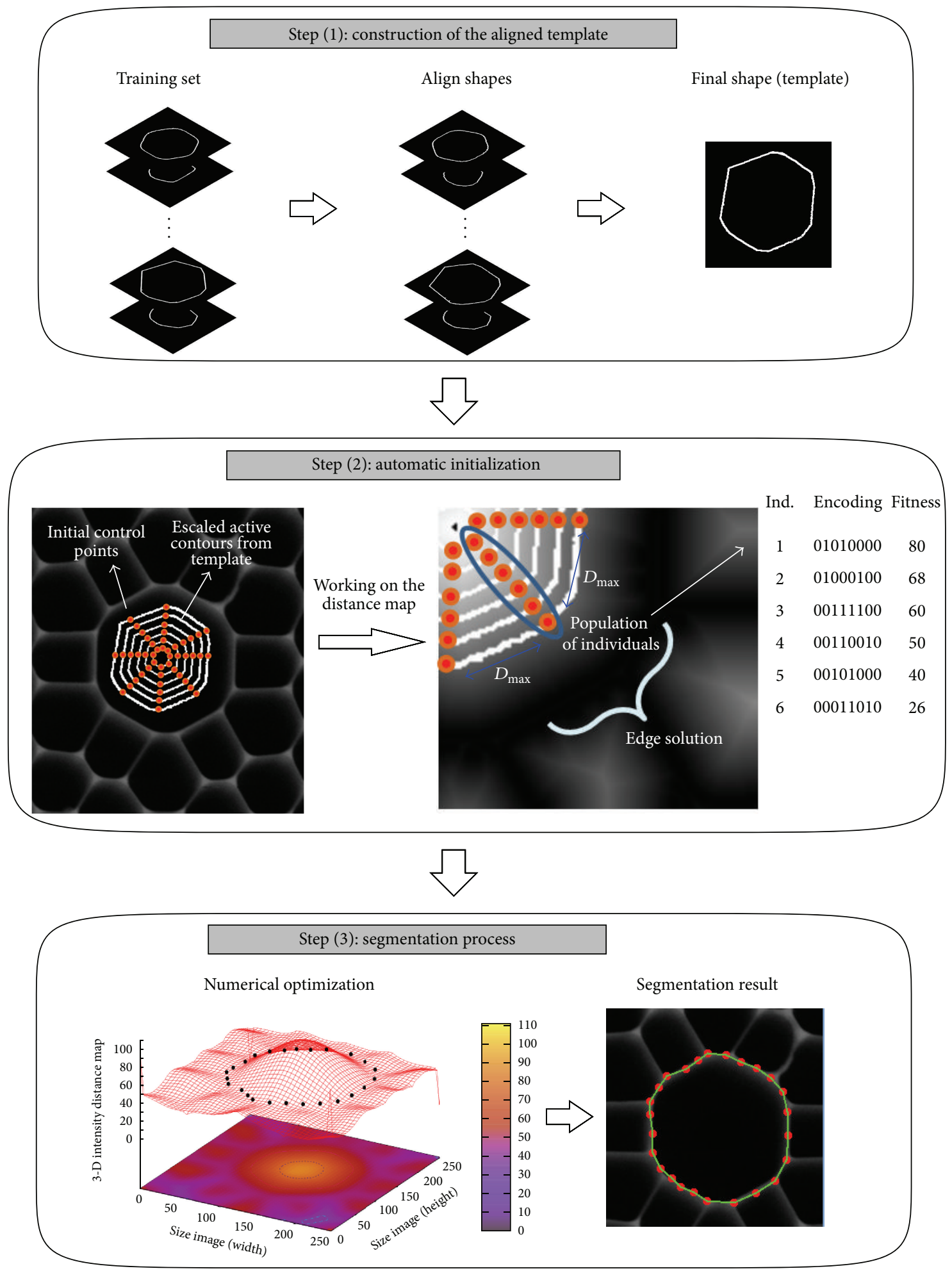

Figure 1: Process of the proposed image segmentation method.

In the second step of the method, a preprocessing stage is required, where we first remove the noise from the test image by using a $2 \mathrm{D}$ median filter $(3 \times 3$ window size), followed by an edge detection between the background and the target object through applying the Canny edge detector, which is experimentally tuned to $\sigma=1.3, T_{l}=10.0$, and $T_{h}=$ 30.0 , in order to preserve the real edges in the test image since these can affect the segmentation result. The final step in this preprocessing, is to compute the Euclidean distance map (EDM) according to [29]. The EDM is used as potential 
surface to perform the optimization process, because it assigns high potential values to the image pixels located far from the target object, and low potential values (ideally zero) to pixels located close to the object. The automatic initialization procedure on the resulting EDM is performed by using the maximum mutual information between the template and the current test image. Subsequently, the $n$ initial active contours are created by scaling the final shape template acquired from the previous alignment process. The number of scaled templates has to be considered assuming that the target object is confined within them. After the $n$ contours are defined, these contours must be discretized by a number $m$ of equidistant control points, this parameter has to be considered to adapt and smooth the segmentation result to the shape of the target object. The control points are assigned as individuals to conform a number $m$ of populations $P$, where each population is composed of individuals of different contours with the same position label. The third step of the proposed method involves the numerical optimization and the image segmentation result. The numerical optimization is performed by using 8 -bit representation (binary encoding) instead of the intensity of the EDM (real encoding in the range $[0,255]$ ) which is used as fitness function in the optimization process. The UMDA strategy is applied for each population $P_{i}$ separately in order to be placed on the nearest edge solution. All the individuals are iteratively evaluated according to the fitness function and the best individual of each population is updated only if a best solution is found considering a maximum distance $D_{\max }$ between best individuals. Finally, when the optimization process for each population $P_{i}$ is finished, the resulting segmented object is acquired by connecting the best individual of each population to each other.

The procedure of the proposed image segmentation method is described as follows.

(1) Align reference shapes according to [22] and obtain final template after alignment.

(2) Perform maximum mutual information to positioning the template.

(3) Initialize number of active contours $n$ and control points $m$.

(4) Initialize the UMDA parameters: number of generations, number of binary bits, and maximum distance $D_{\max }$.

(5) Generate $m$ populations assigning the control points as individuals.

(6) For each population $P_{i}$,

(a) apply restriction of the search space to ignore improper individuals;

(b) evaluate each individual in fitness function derived from the Euclidean distance map;

(c) select a subpopulation of individuals according to selection method;

(d) compute the probabilistic model (univariate marginal distribution); (e) generate $n$ new individuals based on the probabilistic model;

(f) stop if the convergence criterion is satisfied (e.g., stability or number of generations); otherwise go to step (a).

3.2. Evaluation Metrics. To evaluate the performance of the proposed method on medical images, Jaccard and Dice indices have been adopted to analyze the segmentation results between the regions obtained by computational methods and the regions outlined by experts.

The Jaccard index $J(A, B)$ and Dice index $D(A, B)$ are similarity measures used for binary variables and defined in the range $[0,1]$, which are computed using (12) and (13), respectively. In our tests, $A$ represents the reference segmented object outlined by experts and $B$ represents the automatic segmented object by computational methods [12]:

$$
\begin{gathered}
J(A, B)=\frac{A \cap B}{A \cup B}, \\
D(A, B)=\frac{2(A \cap B)}{A+B} .
\end{gathered}
$$

In both indices, when the regions $A$ and $B$ are completely different the obtained result is 0 and is 1 when these two regions are completely superimposed.

In Section 4, the segmentation results obtained from the proposed method on microscopic images of photonic crystal fibers and medical images are presented and analyzed by the evaluation metrics.

\section{Experimental Results}

In this section, the proposed method is applied firstly, on the segmentation of the hollow core in microscopic images of photonic crystal fibers, and secondly, to segment the human heart and ventricular areas from computed tomography and magnetic resonance images. The computational implementations presented in this section are performed using the GNU Compiler Collection $(\mathrm{C}++)$ version 4.4.5 running on Debian GNU/Linux 6.0, Intel Core i3 with $2.13 \mathrm{GHz}$ and $4 \mathrm{~GB}$ of memory.

Figure 2 introduces an image of size $512 \times 512$ pixels consisting of a microscopic image of a hollow core photonic crystal fiber, where the aim is to separate the hollow core from the image. In Figure 2(a) the original test image is presented and its resulting Euclidean distance map is illustrated in Figure 2(b). On the other hand, in Figure 2(c) the segmentation result by using the classical ACM implementation is presented, in which the noise sensitivity and fitting problem are shown. The ACM parameters were statistically determined as 35 control points, $\alpha=0.01, \beta=0.9$, and $\gamma=0.05$ with an executing time of $0.131 \mathrm{~s}$. In Figure 2(d) the segmentation result obtained with the interactive Tseng method is presented. The parameters of this method were set as 35 control points, window size as $30 \times 30$ pixels according to [16], and 9 particles for each swarm, obtaining an executing time of 0.160 s. Finally, in Figure 2(e) the segmented image by 


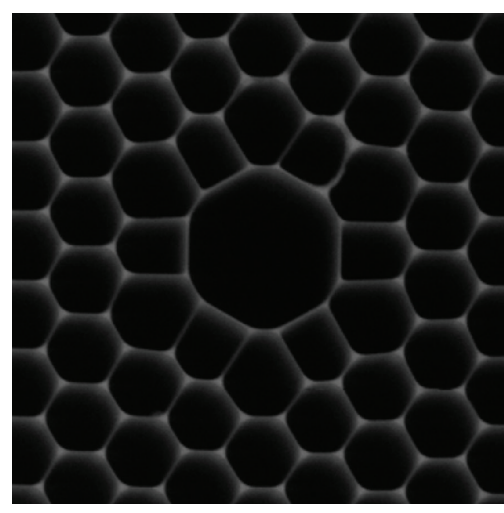

(a)

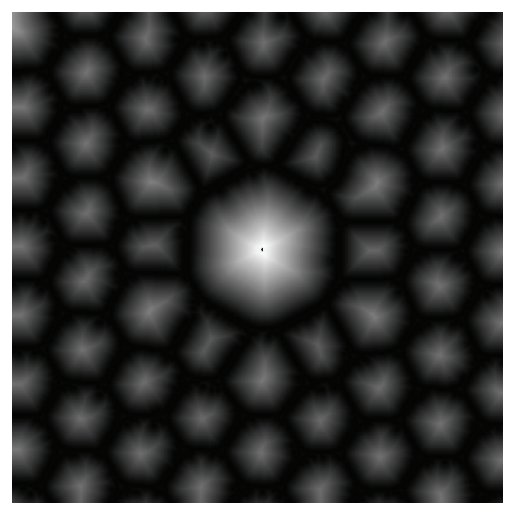

(b)

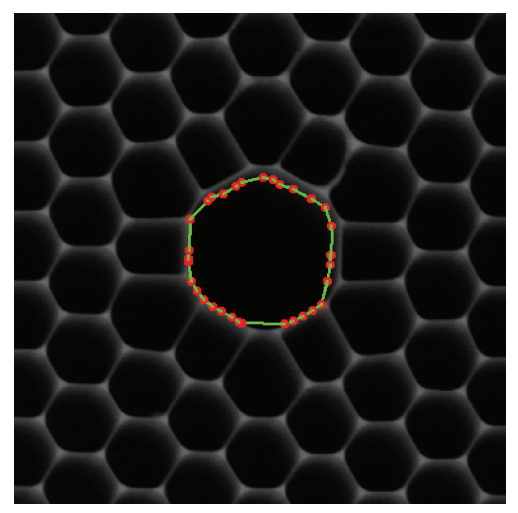

(c)

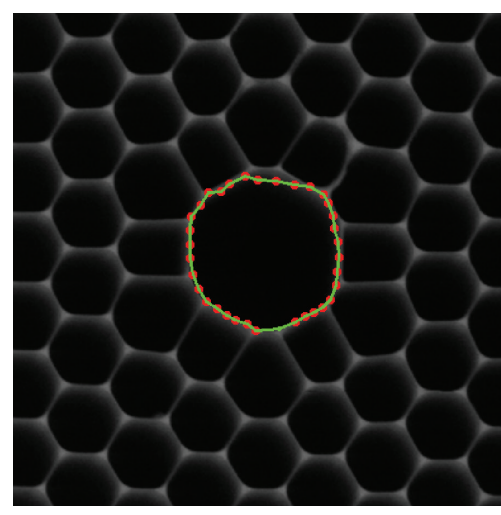

(d)

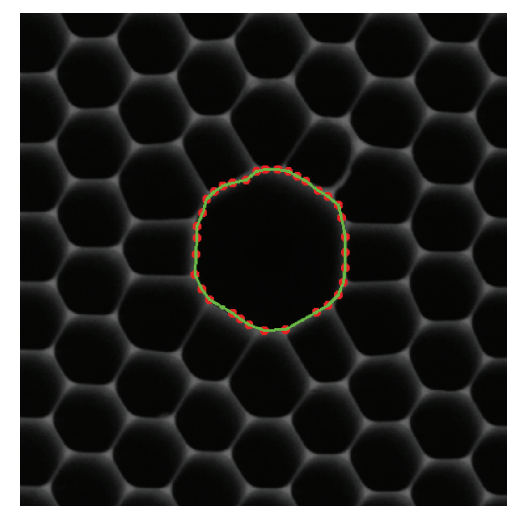

(e)

Figure 2: Hollow core photonic crystal fiber: (a) microscopic test image, (b) Euclidean distance map of test image, (c) segmentation result of classical ACM, (d) segmentation result of interactive Tseng method, and (e) segmentation result of proposed method.

using the proposed method shows an appropriate hollow core segmentation avoiding the local minima problem and locates the hollow core boundary accurately. In this simulation, the parameters were set as generations $=15, D_{\max }=25$, number of contours $=10$, and number of control points $=35$, obtaining an executing time of $0.177 \mathrm{~s}$.

Figure 3 presents a microscopic image of size $512 \times 512$ pixels consisting of another kind of hollow core photonic crystal fiber. In Figures 3(a) and 3(b) the original test image and its resulting Euclidean distance map are illustrated. In Figure 3(c) the segmentation result obtained through the traditional ACM is shown, where due to the noise it cannot adjust to the hollow core boundary accurately. The ACM parameters were experimentally determined as 42 control points, $\alpha=0.013, \beta=0.845$, and $\gamma=0.195$, with an executing time of $0.149 \mathrm{~s}$. In Figure $3(\mathrm{~d})$ the segmentation result by using the interactive Tseng method is presented. The parameters of this simulation were set as 42 control points, window size as $30 \times 30$ pixels, and 9 particles for each swarm, obtaining an executing time of 0.192 s. In addition, Figure 3(e) shows the segmentation result obtained from the proposed method, where it can adjust to the hollow core boundary accurately. The parameters of this experiment were set as generations $=15, D_{\max }=20$, number of contours $=10$, and number of control points $=42$, with an executing time of $0.215 \mathrm{~s}$.
Figure 4 presents the segmentation results on a subset of CT images containing the human heart, where the whole dataset consists of $144 \mathrm{CT}$ images of size $512 \times 512$ pixels obtained from different patients. In Figure 4(a) the human heart outlined by cardiologists is presented. Figure 4(b) illustrates the segmentation results obtained through the classical ACM, where the noise sensitivity and fitting problem are clearly shown. The ACM parameters were determined according to [16] as 45 control points, $\alpha=0.017, \beta=$ 0.86 , and $\gamma=0.45$ with an executing time of $0.161 \mathrm{~s}$. Figure 4(c) illustrates the segmentation results obtained with the interactive Tseng method. The parameters of this simulation were set as 45 control points, window size as $30 \times$ 30 pixels, and 9 particles for each swarm, given an average executing time of 0.205 s per image. Finally, in Figure $4(d)$ the segmentation results obtained by using the proposed method show an appropriate human heart segmentation avoiding the local minima problem. The parameters of this experiment are set as generations $=15, D_{\max }=15$, number of contours $=9$, and number of control points $=45$, with an executing time of $0.209 \mathrm{~s}$.

From the dataset of computed tomography images of the human heart described above, in Table 1 the average of the segmentation results obtained from the classical ACM, interactive Tseng method, and the proposed method is compared with the manual delineations by experts. The comparative 


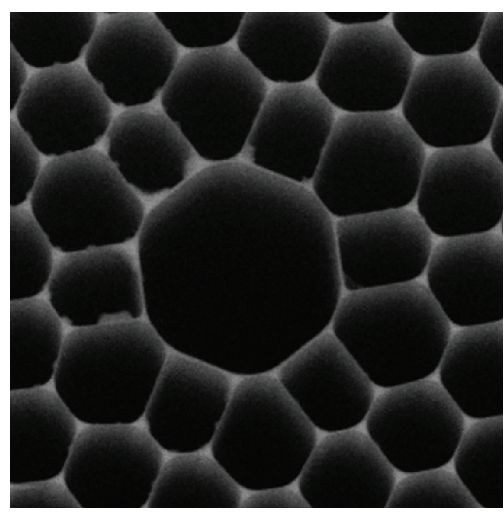

(a)

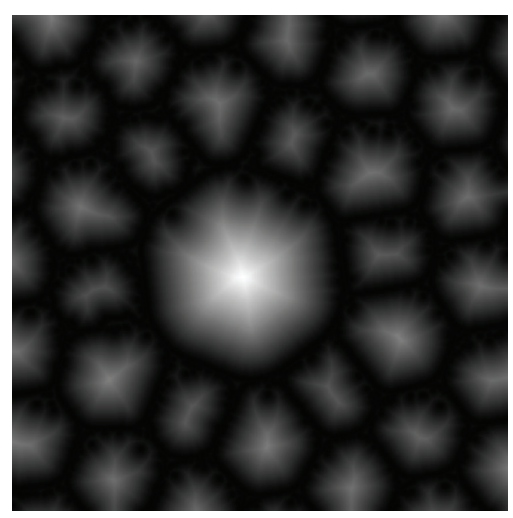

(b)

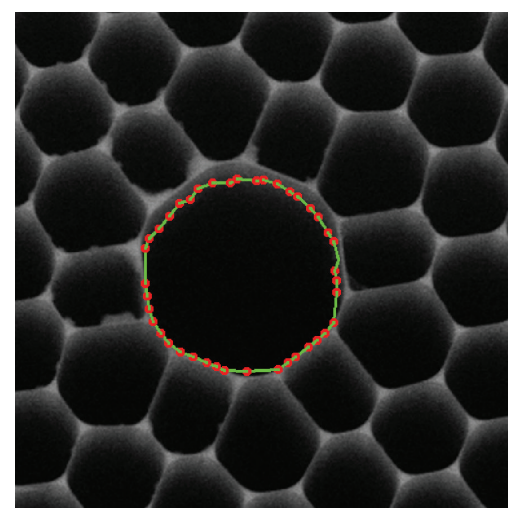

(c)

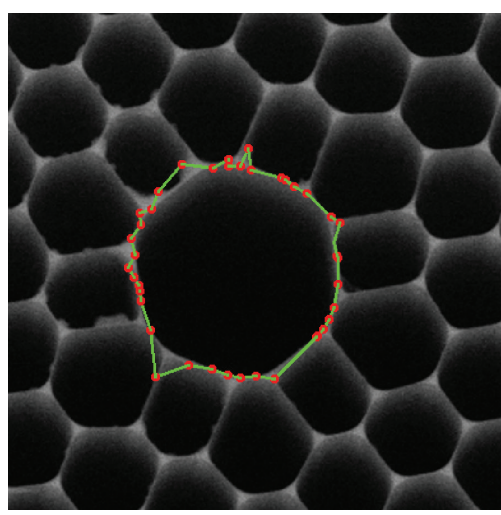

(d)

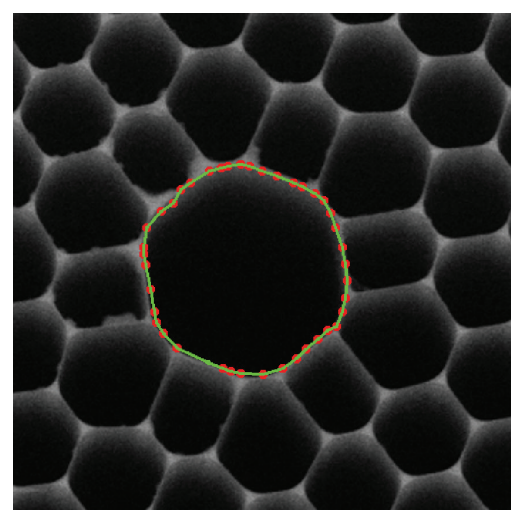

(e)

FIGURE 3: Hollow core photonic crystal fiber: (a) microscopic test image, (b) Euclidean distance map of test image, (c) segmentation result of classical ACM, (d) segmentation result of interactive Tseng method, and (e) segmentation result of proposed method.

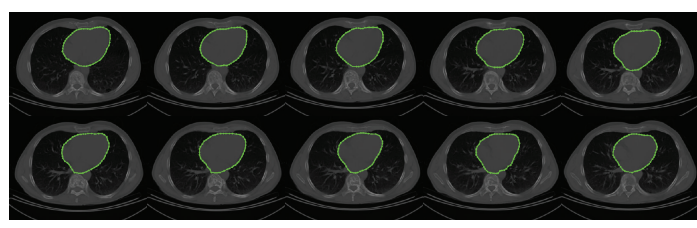

(a)

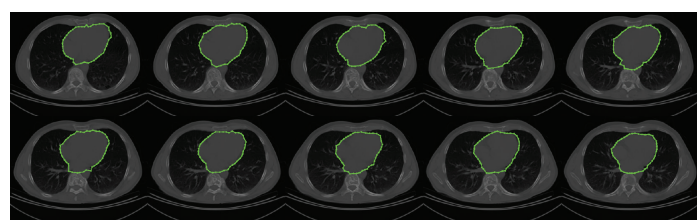

(c)

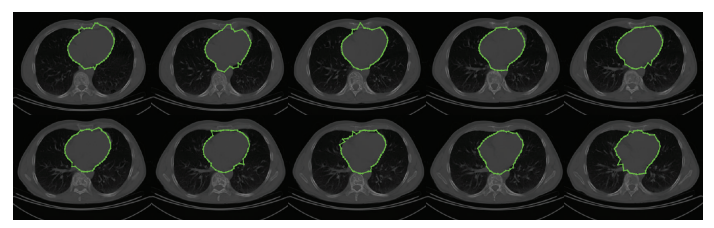

(b)

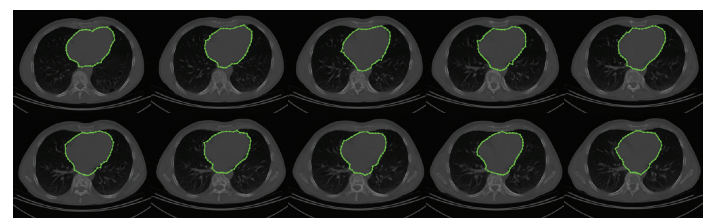

(d)

FIGURE 4: CT images (human heart segmentation): (a) regions outlined by experts, (b) results of classical ACM, (c) results of interactive Tseng method, and (d) segmentation results of proposed method.

analysis reveals that the proposed method is promising for the human heart segmentation on CT images.

In Figure 5 the segmentation results on a subset of magnetic resonance images containing the human ventricular area are presented. The whole set of MR images consists of 19 images with size $256 \times 256$ pixels. In Figure 5(a) human ventricular areas outlined by experts are illustrated. Figure 5(b) presents the segmentation results obtained by the classical ACM, where the method fails to fit the human ventricular area boundary. The ACM parameters were set as 25 control points, $\alpha=0.013, \beta=0.845$, and $\gamma=0.195$, given an average execution time of $0.104 \mathrm{~s}$ per image. In Figure 5(c) the segmentation results through the interactive Tseng method are introduced. In this simulation, the parameters were tuned as 25 control points, window size as $30 \times$ 30 pixels, and 15 particles for each swarm, with an average 


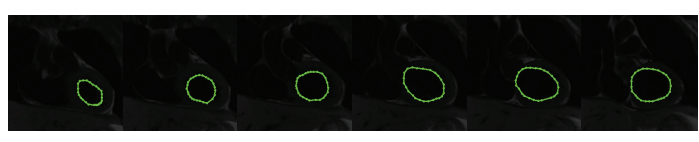

(a)

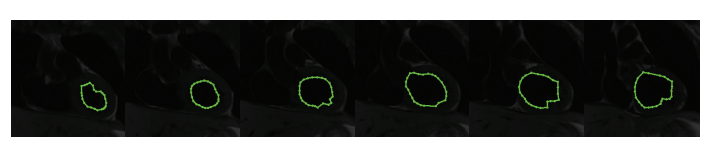

(c)

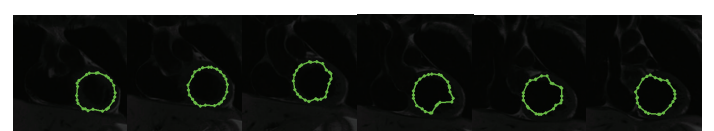

(b)

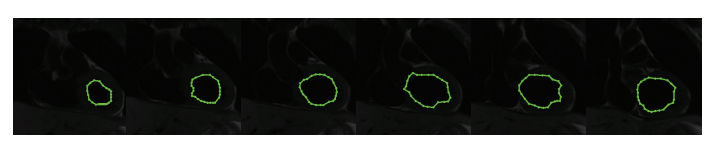

(d)

FIGURE 5: MR images (ventricular area segmentation): (a) regions outlined by experts, (b) results of classical ACM, (c) results of interactive Tseng method, and (d) segmentation results of proposed method.

TABle 1: Average similarity measure with the Jaccard and Dice indices among the regions segmented by the traditional ACM, interactive Tseng method, our proposed method, and the regions outlined by experts of the CT dataset.

\begin{tabular}{lcc}
\hline \multirow{2}{*}{ Comparative studies } & \multicolumn{2}{c}{ Similarity measure } \\
& Jaccard index $(J)$ & Dice index $(D)$ \\
\hline ACM versus experts & 0.6981 & 0.8222 \\
Tseng versus experts & 0.7647 & 0.8666 \\
Our method versus experts & 0.8367 & 0.9111 \\
\hline
\end{tabular}

TABle 2: Average similarity measure with the Jaccard and Dice indices among the ventricular areas segmented by the traditional ACM, interactive Tseng method, our proposed method, and the regions outlined by experts of the MR dataset.

\begin{tabular}{lcc}
\hline \multirow{2}{*}{ Comparative studies } & \multicolumn{2}{c}{ Similarity measure } \\
& Jaccard index $(J)$ & Dice index $(D)$ \\
\hline ACM versus experts & 0.6666 & 0.8000 \\
Tseng versus experts & 0.7857 & 0.8800 \\
Our method versus experts & 0.9230 & 0.9600 \\
\hline
\end{tabular}

executing time of $0.143 \mathrm{~s}$ per image. Moreover, Figure 5(d) shows the segmentation results of the proposed method, where the segmented human ventricular area fit the true boundary accurately. The parameters of this simulation were set as generations $=15, D_{\max }=12$, number of contours $=8$, and number of control points $=25$, with an average executing time of $0.118 \mathrm{~s}$.

According to the human ventricular area dataset of MR images previously described, in Table 2 the average of the segmentation results obtained by the traditional ACM, interactive Tseng method, and our proposed method is compared with the regions outlined by experts. This similarity analysis suggests that the proposed method is more robust in ventricular area segmentation with respect to the comparative computational methods based on the regions outlined by experts.

The use of the Univariate Marginal Distribution Algorithm in the proposed method provides robustness, accuracy and stability in the segmentation problem. Although the computational time of the optimization process is appropriate regarding the comparative computational methods, the proposed image segmentation method improve the segmentation results avoiding the local minima and sensitivity of initialization disadvantages of the classical active contour model.

\section{Conclusions}

In this paper, a novel image segmentation method based on the theory of active contour models and estimation of distribution algorithms has been proposed. This segmentation method has introduced two important advantages with respect to different interactive segmentation techniques: firstly, the automatic initialization by using scaled shape templates obtained from an alignment process, in order to overcome the sensitivity to initial contour position and secondly, the incorporation of statistical information of the control points for addressing the local minima problem. Moreover, this proposed method was applied to segment the hollow core in microscopic images of photonic crystal fibers and it was also used to segment the human heart and ventricular areas from CT and MR images. The experimental results demonstrated that the proposed method can lead to more accuracy and efficiency than the traditional active contour model and the interactive Tseng method. In addition, the experimental results have also shown that the exploitation and exploration capabilities of the proposed method, are highly efficient for different applications according to the evidence showed by the set of similarity measures within a competitive computational time.

\section{Acknowledgments}

This research has been supported by the National Council of Science and Technology of México (CONACYT) under Grant no. 241224-218157. The authors would like to thank for the partial funding provided to this work through the projects: DAIP-UG 01/12 and CONCyTEG GTO-2012-C03195247. The authors wish to thank the cardiology department of the Mexican Social Security Institute UMAE T1 Leon, for the clinical advice and for kindly providing the sources of cardiac CT images. The authors wish to thank the Auckland MRI Research Group, University of Auckland, and Cardiac Atlas Website for the valuable collaboration supplying them the sources of Magnetic Resonance Imaging. 


\section{References}

[1] Y. Boykov and M. Jolly, "Interactive organ segmentation using graph cuts," in Proceedings of the Medical Image Computing and Computer-Assisted Intervention, pp. 276-286, 2000.

[2] F. R. Schmidt, E. Töppe, and D. Cremers, "Efficient planar graph cuts with applications in computer vision," in Proceedings of the IEEE Computer Society Conference on Computer Vision and Pattern Recognition Workshops (CVPR '09), pp. 351-356, June 2009.

[3] W.-Y. Hsu, "Improved watershed transform for tumor segmentation: application to mammogram image compression," Expert Systems with Applications, vol. 39, no. 4, pp. 3950-3955, 2012.

[4] A. Nyma, M. Kang, Y. Kwon, C. Kim, and J. Kim, "A hybrid technique for medical image segmentation," Journal of Biomedicine and Biotechnology, vol. 2012, Article ID 830252, 7 pages, 2012.

[5] M. Guijarro, G. Pajares, I. Riomoros, P. J. Herrera, X. P. BurgosArtizzu, and A. Ribeiro, "Automatic segmentation of relevant textures in agricultural images," Computers and Electronics in Agriculture, vol. 75, no. 1, pp. 75-83, 2011.

[6] E. Cuevas, D. Zaldivar, and M. Pérez-Cisneros, "A novel multi-threshold segmentation approach based on differential evolution optimization," Expert Systems with Applications, vol. 37, no. 7, pp. 5265-5271, 2010.

[7] X. Zhu, P. Zhang, J. Shao, Y. Cheng, Y. Zhang, and J. Bai, "A snake-based method for segmentation of intravascular ultrasound images and its in vivo validation," Ultrasonics, vol. 51, no. 2, pp. 181-189, 2011.

[8] S. Ahmadi, M. J. V. Zoej, H. Ebadi, H. A. Moghaddam, and A. Mohammadzadeh, "Automatic urban building boundary extraction from high resolution aerial images using an innovative model of active contours," International Journal of Applied Earth Observation and Geoinformation, vol. 12, no. 3, pp. 150157, 2010.

[9] B. Wu and Y. Yang, "Local- and global-statistics-based active contour model for image segmentation," Mathematical Problems in Engineering, vol. 2012, Article ID 791958, 16 pages, 2012.

[10] M. Kass, A. Witkin, and D. Terzopoulos, "Snakes: active contour models," International Journal of Computer Vision, vol. 1, no. 4, pp. 321-331, 1988.

[11] T. F. Cootes, C. J. Taylor, D. H. Cooper, and J. Graham, "Active shape models-their training and application," Computer Vision and Image Understanding, vol. 61, no. 1, pp. 38-59, 1995.

[12] J. Hwang, J. Kim, Y. Han, and H. Park, "An automatic cerebellum extraction method in T1-weighted brain MR images using an active contour model with a shape prior," Magnetic Resonance Imaging, vol. 29, no. 7, pp. 1014-1022, 2011.

[13] T. F. Chan and L. A. Vese, "Active contours without edges," IEEE Transactions on Image Processing, vol. 10, no. 2, pp. 266-277, 2001.

[14] M. Talebi, A. Ayatollahi, and A. Kermani, "Medical ultrasound image segmentation using genetic active contour," Journal of Biomedical Science and Engineering, vol. 4, pp. 105-109, 2011.

[15] I. Cruz-Aceves, J. Avina-Cervantes, J. Lopez-Hernandez et al., "Multiple active contours guided by differential evolution for medical image segmentation," Computational and Mathematical Methods in Medicine, vol. 2013, Article ID 190304, 14 pages, 2013.

[16] C.-C. Tseng, J.-G. Hsieh, and J.-H. Jeng, "Active contour model via multi-population particle swarm optimization," Expert Systems with Applications, vol. 36, no. 3, pp. 5348-5352, 2009.
[17] M. Hauschild and M. Pelikan, "An introduction and survey of estimation of distribution algorithms," Swarm and Evolutionary Computation, vol. 1, no. 3, pp. 111-128, 2011.

[18] R. Santana, P. Larrañaga, and J. A. Lozano, "Side chain placement using estimation of distribution algorithms," Artificial Intelligence in Medicine, vol. 39, no. 1, pp. 49-63, 2007.

[19] W. Yan and L. Xiaoxiong, "An improved univariate marginal distribution algorithm for dynamic optimization problem," AASRI Procedia, vol. 1, pp. 166-170, 2012.

[20] A. Petrovski, S. Shakya, and J. McCall, "Optimising cancer chemotherapy using an estimation of distribution algorithm and genetic algorithms," in Proceedings of the 8th Annual Genetic and Evolutionary Computation Conference (GECCO '06), pp. 413-418, July 2006.

[21] R. Shah and P. Reed, "Comparative analysis of multiobjective evolutionary algorithms for random and correlated instances of multiobjective $d$-dimensional knapsack problems," European Journal of Operational Research, vol. 211, no. 3, pp. 466-479, 2011.

[22] A. Tsai, A. Yezzi Jr., W. Wells et al., "A shape-based approach to the segmentation of medical imagery using level sets," IEEE Transactions on Medical Imaging, vol. 22, no. 2, pp. 137-154, 2003.

[23] H. Mühlenbein and G. Paaß, "From recombination of genes to the estimation of distributions i. binary parameters," in Parallel Problem Solving From Nature, pp. 178-187, Springer, 1996.

[24] P. Larrañaga and J. Lozano, Estimation of Distribution Algorithms: A New Tool for Evolutionary Computation, Kluwer Academic, Boston, Mass, USA, 2002.

[25] M. Pelikan, D. E. Goldberg, and F. G. Lobo, "A survey of optimization by building and using probabilistic models," Computational Optimization and Applications, vol. 21, no. 1, pp. 5-20, 2002.

[26] H. Mühlenbein, "The equation for response to selection and its use for prediction," Evolutionary Computation, vol. 5, no. 3, pp. 303-346, 1997.

[27] S. Bashir, M. Naeem, and S. I. Shah, "A comparative study of heuristic algorithms: GA and UMDA in spatially multiplexed communication systems," Engineering Applications of Artificial Intelligence, vol. 23, no. 1, pp. 95-101, 2010.

[28] L.-V. Lozada-Chang and R. Santana, "Univariate marginal distribution algorithm dynamics for a class of parametric functions with unitation constraints," Information Sciences, vol. 181, no. 11, pp. 2340-2355, 2011.

[29] L. D. Cohen and I. Cohen, "Finite-element methods for active contour models and balloons for 2-D and 3-D images," IEEE Transactions on Pattern Analysis and Machine Intelligence, vol. 15, no. 11, pp. 1131-1147, 1993. 


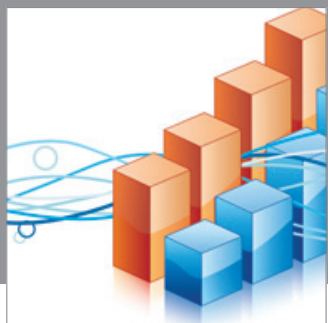

Advances in

Operations Research

mansans

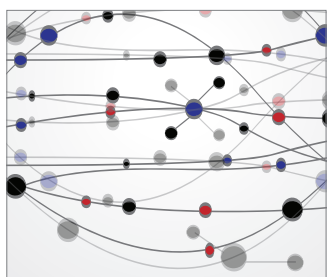

The Scientific World Journal
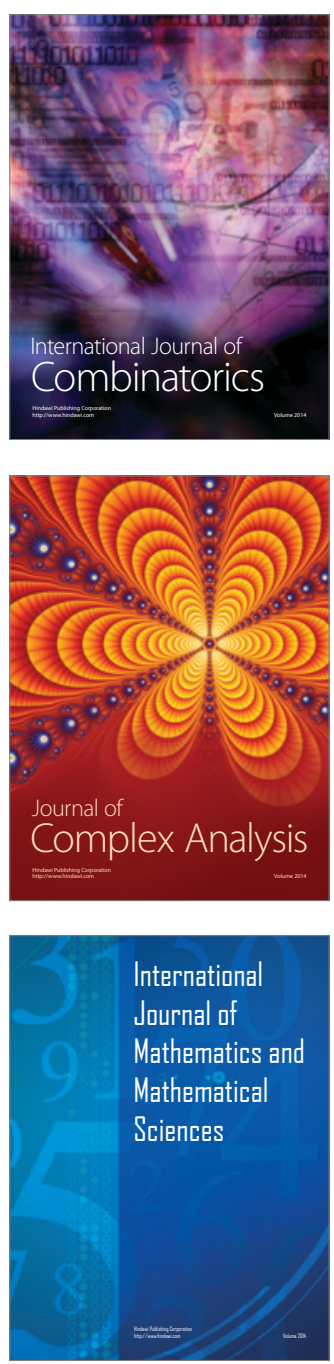
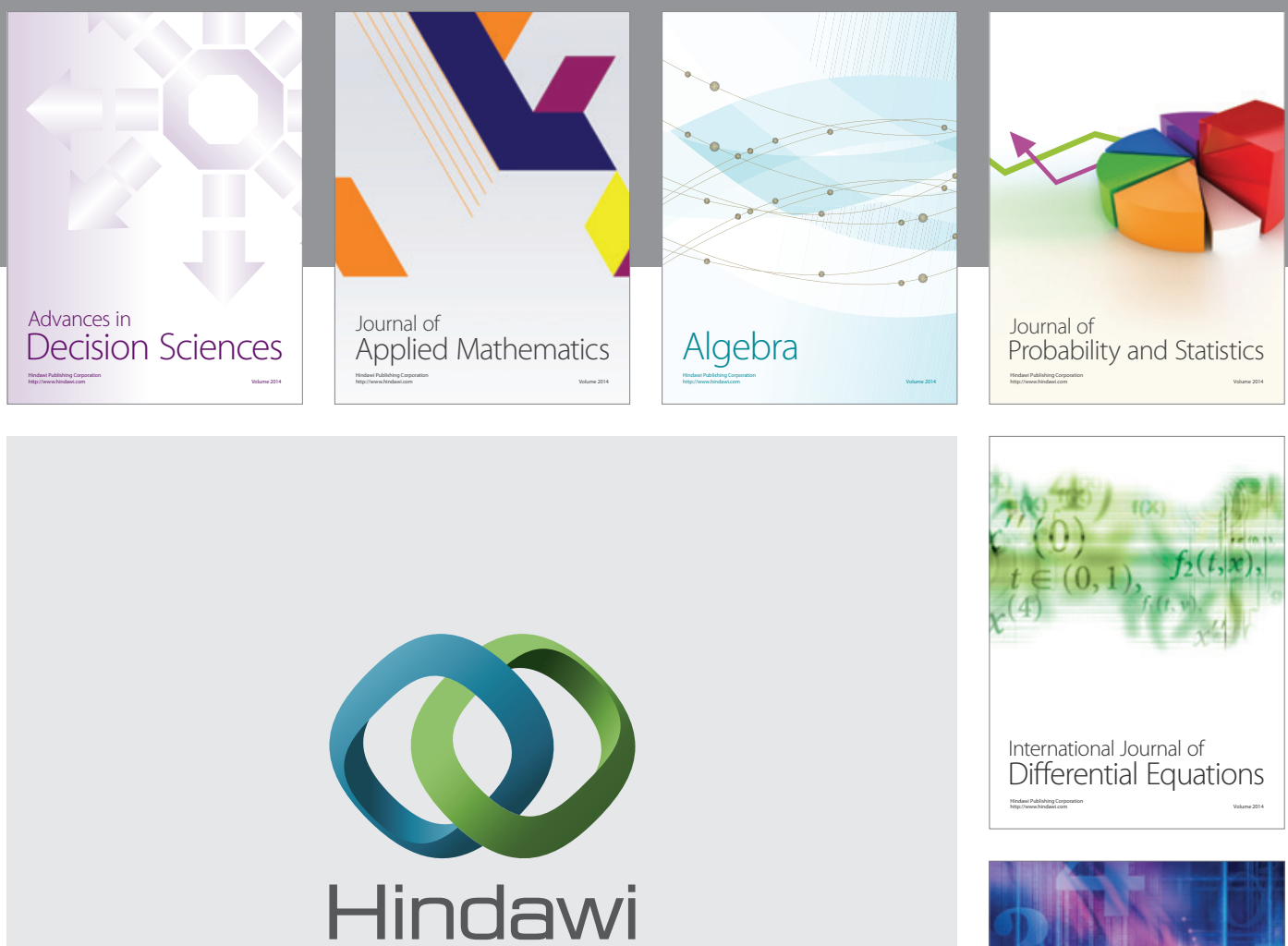

Submit your manuscripts at http://www.hindawi.com
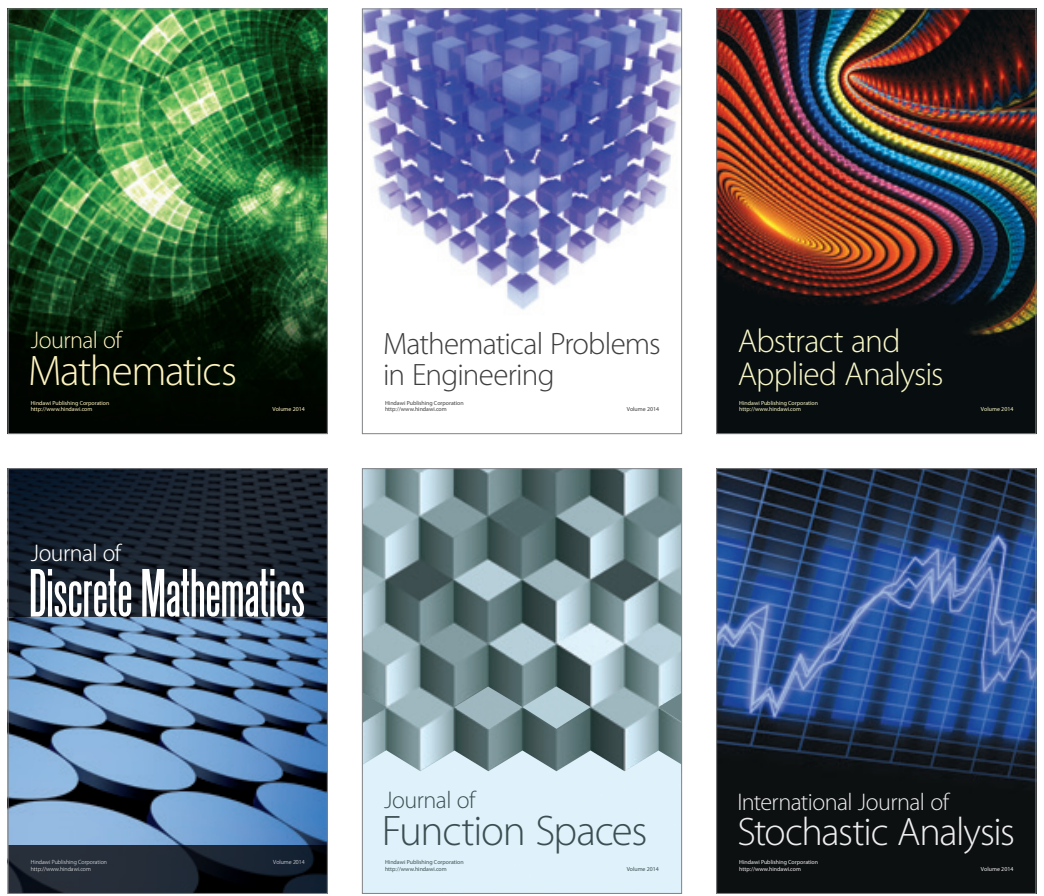

Journal of

Function Spaces

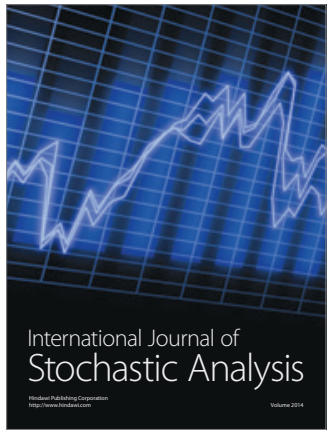

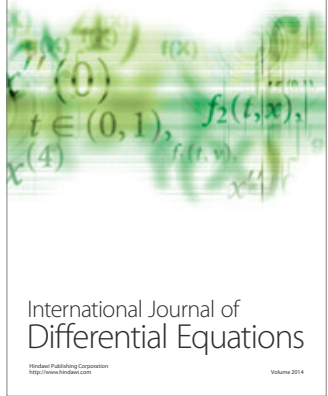
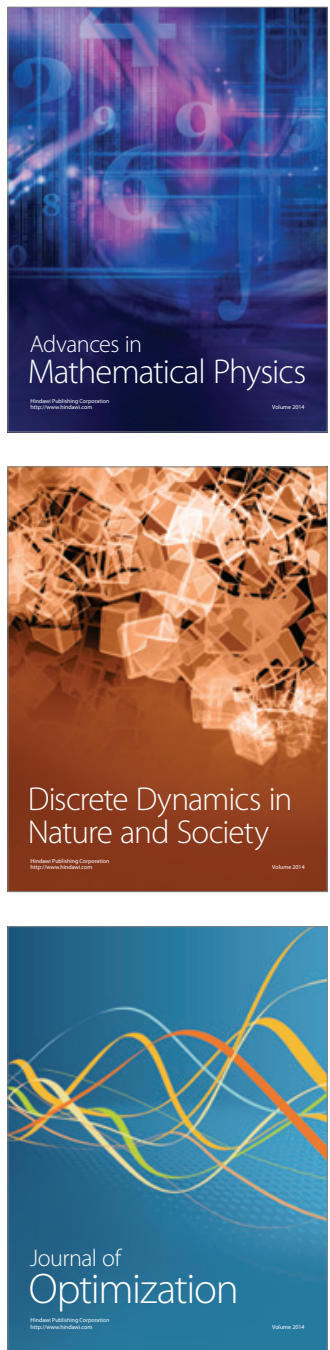